Article

\title{
Periodic Density Functional Calculations in Order to Assess the Cooperativity of the Spin Transition in $\mathrm{Fe}(\mathrm{phen})_{2}(\mathrm{NCS})_{2}$
}

\author{
Hauke Paulsen \\ Institut für Physik, Universität zu Lübeck, Ratzeburger Allee 160, 23562 Lübeck, Germany; \\ paulsen@physik.uni-luebeck.de; Tel.: +49-451-3101-4203; Fax: +49-451-3101-4204 \\ Academic Editors: Guillem Aromí and José Antonio Real \\ Received: 17 January 2016; Accepted: 15 February 2016; Published: 2 March 2016
}

\begin{abstract}
Periodic density functional calculations combined with the Hubbard model $(\mathrm{DFT}+U)$ have been performed for the archetype spin crossover complex Fe(phen $)_{2}(\mathrm{NCS})_{2}$ with phen $=1$,2-phenanthroline. The relative energies of the 16 different configurations of two possible spin states for each of the four molecules in the unit cell have been calculated in order to determine from first principles the phenomenological interaction parameter $\Gamma$ of the Slichter-Drickamer model. These kind of calculations may help to predict important spin crossover characteristics like the abruptness or hysteresis of the transition.
\end{abstract}

Keywords: spin crossover; density functional calculations; Hubbard model; solid state; Slichter-Drickamer model

PACS: 71.15.Mb; 75.30.Wx

\section{Introduction}

A change from an electronic high spin (HS) to a low spin (LS) ground state, commonly called spin crossover (SCO), can be observed in certain transition metal complexes when the temperature is decreased or external pressure is applied. The investigation of the spin crossover phenomenon on its own is a fascinating field of basic research (a renowned review has been edited by Gütlich and Goodwin [1-3]). The discovery that some SCO compounds can be reversibly switched between metastable HS and LS states by irradiation with light (light induced excited spin state trapping, called LIESST effect [4]) made SCO complexes promising materials for memory devices with extremely high capacities.

Spin crossover is an intrinsically molecular phenomenon-even in solutions it is possible to switch the spin state by irradiation with light [5]. However, it is well known that inter-molecular interactions can sensitively influence the character of the spin crossover. For example, the choice of the counterion of a SCO complex could be used to tune the transition temperature $T_{1 / 2}$, i.e., the temperature where half of the complexes are in either spin state. Spin transition curves-as the temperature dependent HS-fraction $\gamma_{\mathrm{HS}}(T)$-exhibit certain features like abruptness, steps or hysteresis only in case of solid samples.

Slichter and Drickamer [6] presented a theoretical model (the so called Slichter-Drickamer model or SD model for short) with only a few empirical parameters, from which many features of the spin transition can be derived. This model belongs to the class of mean field theories since the intermolecular interactions are described by a term that contains only the average magnetization of the crystal (given by the HS-fraction $\gamma_{H S}$ ) and a phenomenological interaction parameter $\Gamma$. The latter is positive if neighboring molecules tend to have the same spin-and negative in the opposite 
case. In a crystal with diluted SCO complexes, and, therefore, no correlation between the spin states of different molecules, $\Gamma$ should vanish. Within the SD model the free enthalpy per molecule can be written as

$$
G\left(\gamma_{\mathrm{HS}}\right)=\gamma_{\mathrm{HS}}\left(\Delta E_{\mathrm{HL}}-T \Delta S_{\mathrm{HL}}+p \Delta V\right)+\gamma_{\mathrm{HS}}\left(1-\gamma_{\mathrm{HS}}\right) \Gamma-T S_{\text {mix }}
$$

where the mixing entropy $S_{\text {mix }}$ is defined by

$$
S_{\text {mix }}=-k_{\mathrm{B}}\left[\gamma_{\mathrm{HS}} \ln \gamma_{\mathrm{HS}}+\left(1-\gamma_{\mathrm{HS}}\right) \ln \left(1-\gamma_{\mathrm{HS}}\right)\right]
$$

and $\Delta E_{\mathrm{HL}}$ and $\Delta S_{\mathrm{HL}}$ are the differences of the total electronic energy and entropy, respectively, between the HS and LS states. The contribution of volume expansion, $p \Delta V$, can be neglected at ambient pressure (usually it amounts to less than $1 \mathrm{~J} \mathrm{~mol}^{-1}$ [7]). The free enthalpy of the pure LS phase has been arbitrarily set to zero: $G(0)=0$. Minimization of $G\left(\gamma_{\mathrm{HS}}\right)$ with respect to $\gamma_{\mathrm{HS}}$ allows to determine the HS-fraction as a function of temperature.

The essential parameters of the SD model- $\Delta E_{\mathrm{HL}}, \Delta S_{\mathrm{HL}}$ and $\Gamma$-may be obtained by fitting experimental data. In this way the SD model may help to interpret and analyze the experiment. If, on the other hand, it would be possible to calculate these parameters from first principles, the SD model might be used to aid the design of new materials, e.g., optical storage devices. Since the pioneering study of Bolvin [8] who investigated the spin state splitting of an $\mathrm{FeN}_{6}$ octahedron with Hartree Fock (HF) calculations a large number of studies have been published which report about electronic structure calculations on SCO complexes (see [7,9-11] for an overview). Wavefunction based methods have the advantage that in principle any order of accuracy can be reached. However, since the spin crossover is the result of a delicate balance of different contributions to the electronic energy, reasonably accurate results for $\Delta E_{\mathrm{HL}}$ can only be reached with sophisticated post-HF methods, which can currently only be applied to systems with a few atoms [12]. For this reason practically all electronic structure calculations on realistic SCO systems have been performed with methods utilizing density functional theory (DFT), either with pure density functionals or with hybrid functionals which include some amount of HF exchange like B3LYP* [13]. In the first years these calculations were restricted to SCO complexes in the gas phase. Since the entropy difference $\Delta S_{\mathrm{HL}}$ is almost purely of molecular origin, this parameter can in some cases be estimated with acceptable accuracy by calculations for the SCO molecule in the gas phase (see for example [14-17]).

The calculation of $\Delta E_{\mathrm{HL}}$ turned out to be more difficult. Tuning the amount of HF exchange in hybrid functionals as well as detailed investigations of the performance of different density functionals with regard to the accuracy of $\Delta E_{\mathrm{HL}}$ [18-26] led to results that are at least in the right order of magnitude. This is especially true if the effect of small ligand modifications should be predicted [27-29]. All methodological progress, however, cannot change the fact that due to inter-molecular interactions the electronic energy difference in the crystal might significantly deviate from the corresponding value for a complex in the gas phase. The phenomenological parameter $\Gamma$ is of course by definition out of the reach of a calculation for a molecule in vacuo.

In order to assess also cooperative effects two approaches have been chosen: The first one is given by molecular calculations for fragments of polymeric SCO materials [30-33] as well as calculations for polynuclear SCO complexes [34-37], whereas the second one consists of electronic structure calculations with periodic boundary conditions. The latter approach was feasible for SCO materials only in the last decade, a first study was published in 2006 by Lemercier et al. [38], who used the local density approximation (LDA) and atom centered basis functions. While part of the periodic DFT studies are concerned with networks of SCO complexes (see for instance [19,39-41]) others are focussed on crystals of monomeric SCO complexes and many of them [12,17,42-45] investigated the complex Fe(phen $)_{2}(\mathrm{NCS})_{2}$ with phen = 1,2-phenanthroline (Figure 1), a complex which-like the majority of the SCO complexes known so far-contains a central iron(II) ion, octahedrally coordinated by six nitrogen atoms. This complex was one of the first SCO complexes discovered at all $[46,47]$ 
and belongs to the SCO complexes most thoroughly studied, experimentally as well as theoretically, and is for this reason often denominated as an archetype SCO complex. It undergoes an abrupt and complete transition from the $S=2 \mathrm{HS}$ state to the $S=0 \mathrm{LS}$ state when the temperature is decreased below the transition temperature $T_{1 / 2} \approx 176 \mathrm{~K}$. The attention this complex has attracted is also due to the fact that it was the first SCO complex for which the crystal structure was available for the HS and for the LS phase [48]. A more recent study by Legrand et al. [49] presents the crystal structure of the LS and of the (metastable) HS phase at $15 \mathrm{~K}$. Fe(phen) $)_{2}(\mathrm{NCS})_{2}$ crystallizes in the space group Pbcn, with four complexes in the unit cell. At $15 \mathrm{~K}$ the volume of the unit cell increases by about $61.3 \AA^{3}$ upon transition from the LS to the HS phase which corresponds to an increase of $2.8 \%$, a typical value for the volume expansion upon spin crossover [1].

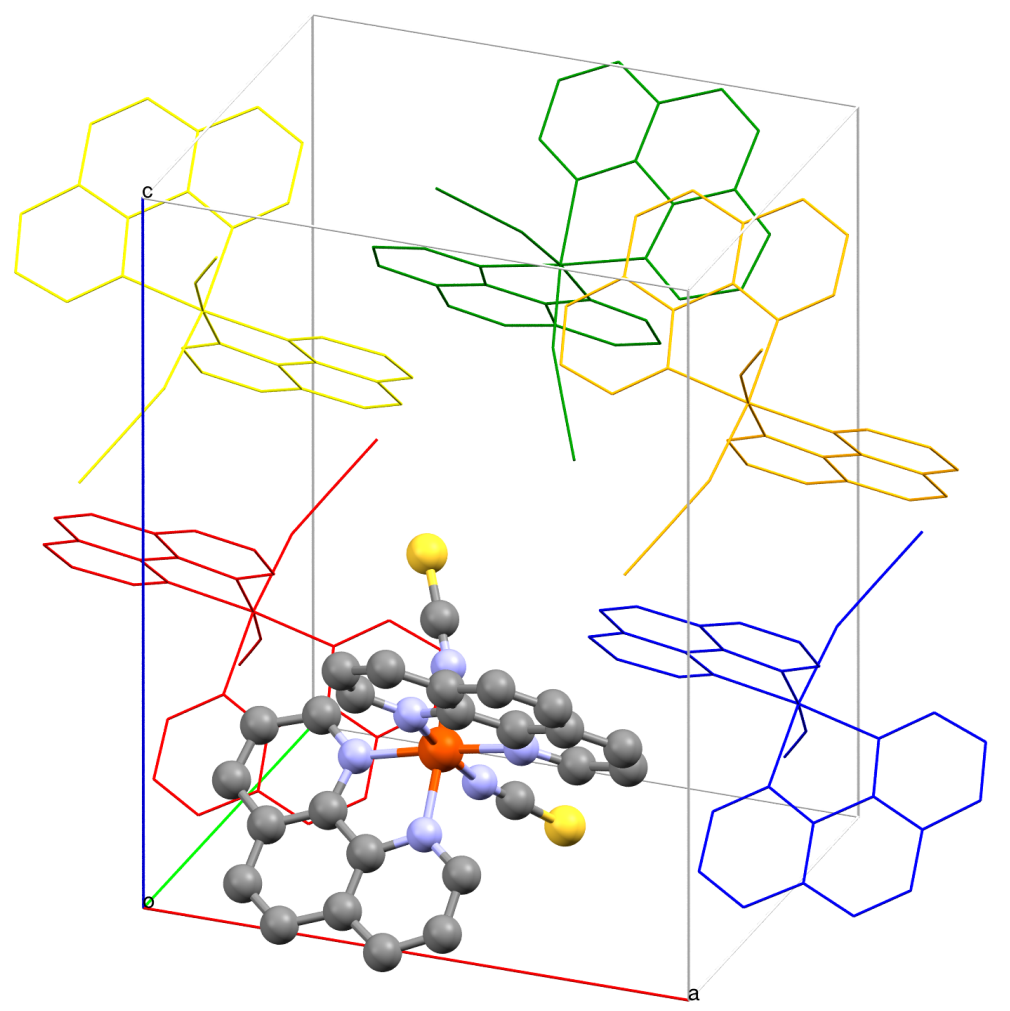

Figure 1. Calculated structure of the low spin (LS) phase of $\mathrm{Fe}(\mathrm{phen})_{2}(\mathrm{NCS})_{2}$ with crystallographic axes and unit cell. One molecule is represented by balls and sticks (color scheme: Fe (red), S (yellow), $\mathrm{N}$ (blue) and C (gray); hydrogens are omitted for clarity), the other molecules are represented by wireframes in different colors. For the two molecules with $x$ coordinates equal to zero for the iron centers also the symmetrically equivalent molecules with $x=1$ are shown, resulting in $Z+2=6$ depicted molecules in total.

$\mathrm{Fe}(\text { phen })_{2}(\mathrm{NCS})_{2}$ is also one of the few SCO complexes where the entropy difference $\Delta S_{\mathrm{HL}}$, which is built up out of two components, an electronic one, $\Delta S_{\mathrm{el}}$, and a vibrational one, $\Delta S_{\mathrm{vib}}$, has been most accurately determined, experimentally as well as theoretically [14-16,50,51]. The electronic component is due to the higher multiplicity of the HS state and can be easily calculated exactly (electronic excitations can be neglected in good approximation), while the vibrational component stems from the fact that loosened metal ligand bonds in the HS state lead to lower vibrational frequencies and-at elevated temperatures-to an increase of entropy. 
While, at least in the case of $\mathrm{Fe}(\mathrm{phen})_{2}(\mathrm{NCS})_{2}, \Delta E_{\mathrm{HL}}$ and $\Delta S_{\mathrm{HL}}$ are well accessible by molecular and periodic electronic structure calculations, $\Gamma$, the parameter describing cooperativity, is more difficult to access. In a recent study from Sinitskiy et al. [52] $\Gamma$ has been derived from atom-atom potentials. In this study a different approach was been chosen. Periodic LDA+ $U$ calculations were performed for different configurations of the spin states of the four molecules of the unit cell of $\mathrm{Fe}(\mathrm{phen})_{2}(\mathrm{NCS})_{2}$ and the resulting energies were used to estimate $\Gamma$ as described below. At zero temperature the entropic terms in this equation vanish and it can be rewritten as

$$
E\left(\gamma_{\mathrm{HS}}, c\right)=\gamma_{\mathrm{HS}} \Delta E_{H L}+\gamma_{\mathrm{HS}}\left(1-\gamma_{\mathrm{HS}}\right) \Gamma(c)
$$

where the total electronic energy per molecule in the pure LS phase $\left(\gamma_{\mathrm{HS}}=0\right)$ has been arbitrarily set to zero. The index $c$ denotes the configuration of the crystal with respect to the spin states (HS or LS) of the individual SCO molecules. In general, for a crystal with $N$ SCO molecules there are $2^{N}$ possible configurations and the HS-fraction $\gamma_{\mathrm{HS}}$ could be written as a function of the configuration $c$. While there is only one configuration that corresponds to the pure LS phase $\left(\gamma_{\mathrm{HS}}=0\right)$ and the same is valid for the pure HS phase, there are $\left(\begin{array}{c}N \\ N / 2\end{array}\right)$ different configurations for the critical HS-fraction $\gamma_{\mathrm{HS}}=1 / 2$. In the latter case not all configurations $c$ are energetically favorable to the same degree and the electronic energy $E\left(\gamma_{\mathrm{HS}}, c\right)$ is a function of $c$. Since the HS-fraction $\gamma_{H S}(c)$ is a function of $c$ too, we can omit $\gamma_{\mathrm{HS}}$ as an argument and write the electronic energy shortly as $E(c)$. Since the left side of Equation (3) is varying with the configuration $c$ even at constant $\gamma_{\mathrm{HS}}$, the same must be true for the right side of this equation resulting in a configuration dependent parameter

$$
\Gamma(c)=\frac{E(c)-\gamma_{\mathrm{HS}} \Delta E_{\mathrm{HL}}}{\gamma_{\mathrm{HS}}\left(1-\gamma_{\mathrm{HS}}\right)}
$$

for configurations $c$ with $0<\gamma_{\mathrm{HS}}<1$ (if $\gamma_{\mathrm{HS}}=0$ or $\gamma_{\mathrm{HS}}=1$ the term $\gamma_{\mathrm{HS}}\left(\gamma_{\mathrm{HS}}-1\right) \Gamma$ in Equations (1) and (3) vanishes and $\Gamma$ becomes irrelevant). The macroscopic interaction parameter $\Gamma$ that is used in the SD model as defined in Equation (1) can now be written as the weighted sum

$$
\Gamma=\sum_{c} w(c) \Gamma(c)
$$

where the weights $w(c)=f(c) / Z$ are given by the Boltzmann factor

$$
f(c)=\exp \left(-\frac{E(c)-\gamma_{\mathrm{HS}} \Delta E_{\mathrm{HL}}}{k_{\mathrm{B}} T_{1 / 2}}\right)
$$

normalized by the partition function $Z=\sum_{c} f(c)$ (the numerator of the fraction in the preceding equation reflects the fact that at the critical temperature both spin states are in equilibrium and the Boltzmann weight of the pure LS phase should be the same as the one of the pure HS phase). For sufficiently steep transitions it is well justified to replace the temperature $T$ in the exponent of the Boltzmann factor by the critical temperature $T_{1 / 2}$ as it is done here, since we have $\gamma_{\mathrm{HS}} \approx 0$ for $T \ll T_{1 / 2}$ and $\gamma_{\mathrm{HS}} \approx 1$ for $T \gg T_{1 / 2}$ (in case of a pronounced gradual transition the temperature $T$ has to be written as a function of the HS-fraction leading to a system of equations that has to be solved self consistently). In this way the cooperativity of a SCO complex can be predicted by first principles calculations without need of empirical potentials like in the approach from Sinitskiy et al. [52]. The disadvantage however is, that the approach presented here is computationally much more demanding and for this reason restricted to the unit cell or at maximum to small super cells, which might reduce the accuracy. 


\section{Results and Discussion}

From variable cell geometry optimization with $U=2.5,4.0,5.6$ and $6.0 \mathrm{eV}$ for the pure LS and the pure HS phase an almost perfectly linear dependence between $\Delta E_{\mathrm{HL}}$ and $U$ was obtained. For $U=5.6 \mathrm{eV}$ the total energy difference amounts to $\Delta E_{\mathrm{HL}} \approx 9.77 \mathrm{~kJ} \mathrm{~mol}^{-1}$ fitting well to the experimentally determined $9 \mathrm{~kJ} \mathrm{~mol}^{-1}$ from Sorai and Seki [53]. A similar linear dependence between $\Delta E_{\mathrm{HL}}$ and $U$ was found after including the Grimme-D2 dispersion correction [54]. The obtained Hubbard $U$ of $5.6 \mathrm{eV}$ is at the upper limit of the range of values $(2.5$ to $5.0 \mathrm{eV})$ that were reported $[12,17,42-45]$ for this parameter in case of calculations for $\mathrm{Fe}(\mathrm{phen})_{2}(\mathrm{NCS})_{2}$. However, all studies on this complex that employed a small Hubbard parameter of about $2.5 \mathrm{eV}[12,17,42]$ were performed with gradient corrected density functionals which should better account for the localization of the strongly correlated iron $3 \mathrm{~d}$ electrons and thus have less need for the Hubbard correction.

The volume expansion upon complete spin transition was calculated to $65 \AA^{3}$ and $76 \AA^{3}$ with and without Grimme-D2 correction, respectively, which is in reasonable agreement with the experimental result of $79 \AA^{3}$ [49]. However, with dispersion correction the volume of the HS unit cell results to $1832 \AA^{3}$, whereas without this correction $1996 \AA^{3}$ were obtained which is closer to the experimental value of $2248 \AA^{3}$. This finding might be traced back to the notorious underestimation of bond lengths by LDA methods [55]. Since dispersion corrections usually lead to smaller calculated volumes, neglecting dispersion in LDA calculations might lead to a partial cancellation of errors with respect to the volume. The calculations discussed in the following were performed without dispersion correction.

In order to assess the cooperativity of spin transitions using first principles methods the 16 possible spin configurations of the four molecules in the unit cell of $\mathrm{Fe}(\mathrm{phen})_{2}(\mathrm{NCS})_{2}$ have been studied (see also Figure 2). For each configuration $c$ (labeled with a four letter code like LLHL for a configuration with three LS molecules and one HS molecule in a given order) a complete variable-cell geometry optimization has been performed. The resulting total energies per molecule (Table 1) clearly do not depend linearly on the HS-fraction $\gamma_{\mathrm{HS}}$ (in other words the number of HS molecules divided by the number of molecules in the cell). If the energy needed to switch a molecule from the LS to the HS state would be independent from the spin state of the neighboring molecules, the total energy per molecule of configurations with $\gamma_{\mathrm{HS}}=0.5$ (like LLHH or LHLH where the four letters stand for the spin states of the four molecules of the unit cell, L indicating a molecule in the low spin state and $\mathrm{H}$ indicating a molecule in the high spin state) should be $\Delta E_{\mathrm{HL}} / 2 \approx 4.9 \mathrm{~kJ} \mathrm{~mol}^{-1}$. Instead we found significantly larger values of in average $7.0 \mathrm{~kJ} \mathrm{~mol}^{-1}$, indicating that different spin states for neighboring molecules are energetically not favorable. In the SD model this should be reflected by a positive parameter $\Gamma$.

For the four configurations that correspond to $\gamma_{\mathrm{HS}}=0.75$ (the two rightmost columns in Table 1) as well as for the configurations LHLH and HLHL (called group 1 in the following ) values for $\Gamma(c)$ are obtained with the help of Equation (4) that range from 4.9 to $5.7 \mathrm{~kJ} \mathrm{~mol}^{-1}$ as compared to values between 8.5 and $12.1 \mathrm{~kJ} \mathrm{~mol}^{-1}$ for the remaining eight configurations (group 2). The configurations of group 1 have in common that one yz-plane of the unit cell is filled with HS molecules and that, therefore, the size of the unit cell is close to the size in the pure HS phase. In this way there is sufficient space for the HS molecules and the electronic energy per HS molecule is in average $11.6 \mathrm{~kJ} \mathrm{~mol}^{-1}$, roughly $1.8 \mathrm{~kJ} \mathrm{~mol}^{-1}$ more than $\Delta E_{\mathrm{HL}}$. In each configuration of group 2 on the other hand there is at least one LS molecule in each of the two $y z$-planes of the unit cell and the volume of the cell is less than in the pure HS phase. Therefore, there is insufficient space for the HS molecules and their electronic energy is in average $16.6 \mathrm{~kJ} \mathrm{~mol}^{-1}$, which is about $6.8 \mathrm{~kJ} \mathrm{~mol}^{-1}$ more than $\Delta E_{\mathrm{HL}}$. As a result the correlation between the spin states of neighboring molecules is less in group 1 than in group 2 (no correlation would correspond to an electronic energy per HS molecule of exactly $\Delta E_{\mathrm{HL}}$ ) and consequently the interaction parameter $\Gamma(c)$ for the configurations of group 1 is smaller than for the configurations of group 2. 


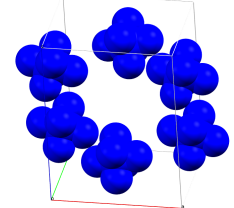

(a) LLLL

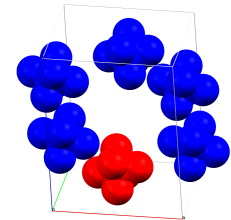

(b) HLLL

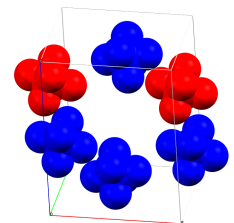

(c) LHLL

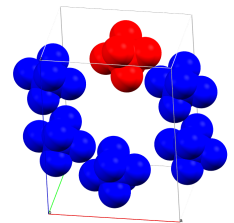

(d) LLHL

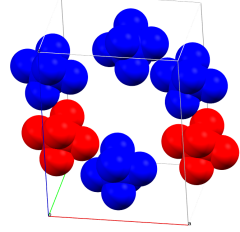

(e) LLLH

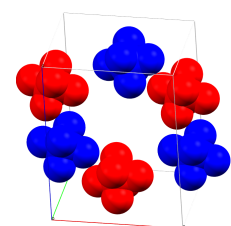

(f) HHLL

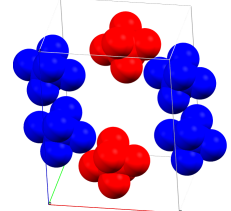

(g) HLHL

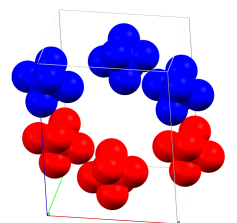

(h) HLLH

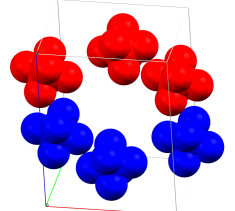

(i) LHHL

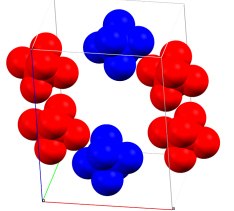

(j) $\mathrm{LHLH}$

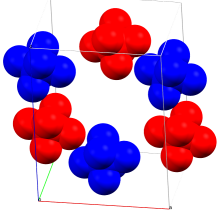

(k) $\mathrm{LLHH}$

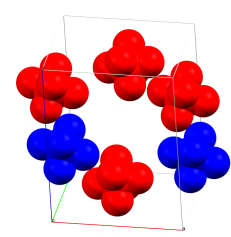

(l) HHHL

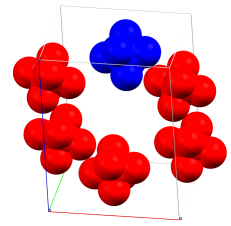

(m) HHLH

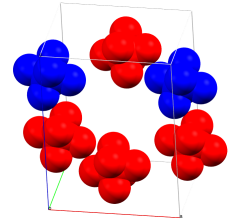

(n) HLHH

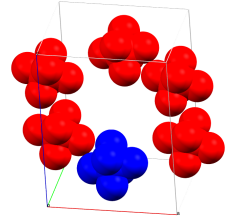

(o) $\mathrm{LHHH}$

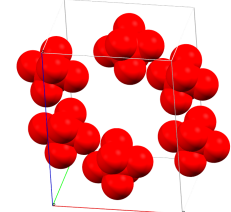

(p) $\mathrm{HHHH}$

Figure 2. 16 different spin configurations of a unit cell: (a) one configuration with $\gamma_{\mathrm{HS}}=0$; (b)-(e) four configurations with $\gamma_{\mathrm{HS}}=0.25 ;(\mathbf{f})-(\mathbf{k})$ six configurations with $\gamma_{\mathrm{HS}}=0.5 ;(\mathbf{l})-(\mathbf{o})$ four configurations with $\gamma_{\mathrm{HS}}=0.75$ and (p) one configuration with $\gamma_{\mathrm{HS}}=1$. Low spin (LS) and high spin (HS) molecules are colored in blue and red, respectively, and only the central $\mathrm{FeN}_{6}$ octahedron is shown. As in Figure $2 \mathrm{Z}+2$ molecules are shown in total for each configuration. The four letter codes (like LLHH or LHLH) stand for the spin states of the four molecules of the unit cell, L indicating a molecule in the low spin state and $\mathrm{H}$ indicating a molecule in the high spin state. 
Table 1. Total electronic energies per molecule, $E(c)$, in $\mathrm{kJ} \mathrm{mol}^{-1}$ for different spin configurations $c$ given by four letter codes (like LLHH or LHLH) as defined in Figure 2. The energy of the LLLL configuration $\left(\gamma_{\mathrm{HS}}=0\right)$ is set to zero.

\begin{tabular}{cccccccc}
\hline \multicolumn{1}{c}{$c$} & $\boldsymbol{E}(\boldsymbol{c})$ & $\boldsymbol{c}$ & $\boldsymbol{E}(\boldsymbol{c})$ & $\boldsymbol{c}$ & $\boldsymbol{E}(\boldsymbol{c})$ & $\boldsymbol{c}$ & $\boldsymbol{E}(\boldsymbol{c})$ \\
\hline HLLL & 4.71 & HHLL & 7.55 & HHHL & 8.33 & HHHH & 9.77 \\
LHLL & 4.40 & HLHL & 6.20 & HHLH & 8.30 & & \\
LLHL & 4.61 & HLLH & 7.29 & HLHH & 8.24 & & \\
LLLH & 4.60 & LHHL & 7.71 & LHHH & 8.38 & & \\
& & LHLH & 6.31 & & & & \\
& & LLHH & 7.00 & & & & \\
\hline
\end{tabular}

As an unweighted average value we would get $\Gamma \approx 8.4 \mathrm{~kJ} \mathrm{~mol}^{-1}$, which is more than twice as large as the experimental value of $3.0 \mathrm{~kJ} \mathrm{~mol}^{-1}$ from Sinitskiy et al. [52] but in the same order of magnitude. A weighted average according to Equation (5) gives a value of $7.3 \mathrm{~kJ} \mathrm{~mol}^{-1}$ that fits somewhat better to the experimental value but is still more than twice as large. One quite obvious reason for this deviation is the small cell with only four SCO molecules that has been used for the present study. Studies that employ the Ising model together with Monte Carlo simulations on large supercells indicate that large clusters of equal spins are energetically favorable to configurations with neighboring molecules with different spin states [56]. Finally, the $\Gamma$ parameter predicted by Sinitskiy et al. with a completely different theoretical approach (between 5 and $8 \mathrm{~kJ} \mathrm{~mol}^{-1}$ ) is larger than the experimental value as well-thus leaving room for the speculation that the SD model does not perfectly describe the spin transition of $\mathrm{Fe}(\mathrm{phen})_{2}(\mathrm{NCS})_{2}$. A possible reason for this may be the influence of inter- and intra-molecular mode coupling on the cooperative behavior of the spin transition observed by Mebs et al. [57].

\section{Computational Details}

All calculations were performed with the software suite Quantum ESPRESSO (Version 5.2.0, QE Foundation, Trieste, Italy, 2015) [58]. Valence electrons were represented explicitly by plane waves with cutoffs of $50 \mathrm{Ry}$ for the waves and $400 \mathrm{Ry}$ for the charge density. Self consistent wavefunctions and forces were converged to an accuracy of $10^{-7}$ and $10^{-5}$ atomic units, respectively. Less strict cutoffs for the forces influenced adversely the accuracy of the calculated interaction parameter $\Gamma$. The Brillouin zone sampling was restricted to the $\Gamma$ point. The interactions between the valence electrons were described within the local density approximation with the help of the exchange and correlation functional from Perdew and Zunger [59]. Ultrasoft pseudopotentials [60] were employed [61] to treat the core electrons implicitly by replacing the all-electron Hamiltonian by a pseudo-Hamiltonian that describes the interactions between the ions and the valence electrons. In order to describe better the localization of the strongly correlated iron $3 \mathrm{~d}$ electrons the DFT $+U$ model with the simplified scheme of Cococcioni et al. [62] was used with a Hubbard $U$ of $5.6 \mathrm{eV}$ if not explicitly stated otherwise. For some calculations in addition the Grimme-D2 correction [54] was applied to include the influence of the otherwise poorly described London forces.

Since the electronic structure calculations were performed for the state at zero temperature the X-ray structures from Legrand et al. [49] was used as starting points, where the structure of the LS- and of the metastable HS-phase have been determined at $T=15 \mathrm{~K}$.

\section{Conclusions}

From first principles calculations for different spin configurations of the unit cell of a SCO complex a reasonable estimate for the phenomenological interaction parameter $\Gamma$ was obtained that allows to describe the cooperativity of the spin transition within the SD model. Future calculations will have to show whether a better agreement between the theoretical and the experimental value for $\Gamma$ can be reached, for instance by choosing a gradient corrected density functional together 
with a dispersion correction or by performing calculations for supercells. In any case such kind of calculations might help to predict important characteristics like the abruptness and the hysteresis of the spin transition. Beyond the validity of the SD model the type of calculations presented here may also be useful to deliver input parameters to more sophisticated models that describe the spin transition in SCO materials.

Acknowledgments: This article is dedicated to the 75th birthday of Alfred X. Trautwein.

Conflicts of Interest: The authors declare no conflict of interest.

\section{References}

1. Gütlich, P., Goodwin, H.A., Eds.; Spin Crossover in Transition Metal Compounds I, 1st ed.; Topics in Current Chemistry; Springer: Berlin, Germany; Heidelberg, Germany; New York, NY, USA, 2004; Volume 1.

2. Gütlich, P., Goodwin, H.A., Eds.; Spin Crossover in Transition Metal Compounds II, 1st ed.; Topics in Current Chemistry; Springer: Berlin, Germany; Heidelberg, Germany; New York, NY, USA, 2004; Volume 2.

3. Gütlich, P., Goodwin, H.A., Eds.; Spin Crossover in Transition Metal Compounds III, 1st ed.; Topics in Current Chemistry; Springer: Berlin, Germany; Heidelberg, Germany; New York, NY, USA, 2004; Volume 3.

4. Decurtins, S.; Gütlich, P.; Köhler, C.; Spiering, H.; Hauser, A. Light-induced excited spin state trapping in a transition-metal complex: The hexa-1-propyltetrazole-iron (II) tetrafluoroborate spin-crossover system. Chem. Phys. Lett. 1984, 139, 1-4.

5. McGarvey, J.J.; Lawthers, I. Photochemically-induced perturbation of the ${ }^{1} \mathrm{~A} \rightleftharpoons{ }^{5} \mathrm{~T}$ equilibrium in $\mathrm{Fe}^{\mathrm{II}}$ complexes by pulsed laser irradiation in the metal-to-ligand charge-transfer absorption band. J. Chem. Soc. Chem. Commun. 1982, doi:10.1039/C39820000906.

6. Slichter, C.P.; Drickamer, H.G. Pressure-induced electronic changes in compounds of iron. J. Chem. Phys. 1972, 56, 2142-2160.

7. Paulsen, H.; Trautwein, A. Density functional theory calculations for spin crossover complexes. Top. Curr. Chem. 2004, 235, 197-219.

8. Bolvin, H. d->d spectrum and high-spin/low-spin competition in $\mathrm{d}^{6}$ octahedral coordination compounds: Ab initio study of potential energy curves. J. Phys. Chem. A 1998, 102, 7525-7534.

9. Paulsen, H.; Schünemann, V.; Wolny, J.A. Progress in electronic structure calculations on spin-crossover complexes. Eur. J. Inorg. Chem. 2013, doi:10.1002/ejic.201201289.

10. Kepp, K.P. Consistent description of metal-ligand bonds and spin-crossover in inorganic chemistry. Coord. Chem. Rev. 2013, 257, 196-209.

11. Deeth, R.J. Molecular discovery in spin crossover. In Spin States in Biochemistry and Inorganic Chemistry; John Wiley \& Sons, Ltd.: Chichester, United Kingdom, 2015; pp. 85-102.

12. Vela, S.; Fumanal, M.; Ribas-Arino, J.; Robert, V. Towards an accurate and computationally-efficient modelling of Fe(II)-based spin crossover materials. Phys. Chem. Chem. Phys. 2015, 17, 16306-16314.

13. Reiher, M.; Salomon, O.; Hess, B.A. Reparameterization of hybrid functionals based on energy differences of state of different multiplicity. Theor. Chem. Acc. 2001, 107, 48-55.

14. Reiher, M. Theoretical study of the Fe(phen $)_{2}(\mathrm{NCS})_{2}$ spin-crossover complex with reparametrized density functionals. Inorg. Chem. 2002, 41, 6928-6935.

15. Reiher, M.; Brehm, G.; Schneider, S. Assignment of vibrational spectra of 1,10-phenanthroline by comparison with frequencies and raman intensities from density functional calculations. J. Phys. Chem. A 2004, 108, 734-742.

16. Ronayne, K.L.; Paulsen, H.; Höfer, A.; Dennis, A.C.; Wolny, J.A.; Chumakov, A.I.; Schünemann, V.; Winkler, H.; Spiering, H.; Bousseksou, A.; et al. Vibrational spectrum of the spin-crossover complex $\left[\mathrm{Fe}(\text { phen })_{2}(\mathrm{NCS})_{2}\right]$ studied by IR and Raman spectroscopy, nuclear inelastic scattering and DFT calculations. Phys. Chem. Chem. Phys. 2006, 8, 4685-4693.

17. Bučko, T.; Hafner, J.; Lebègue, S.; Ángyán, J.G. Spin crossover transition of Fe(phen $)_{2}(\mathrm{NCS})_{2}$ : Periodic dispersion-corrected density-functional study. Phys. Chem. Chem. Phys. 2012, 14, 5389-5396.

18. Jensen, K.P.; Cirera, J. Accurate computed enthalpies of spin crossover in iron and cobalt complexes. J. Phys. Chem. A 2009, 113, 10033-10039. 
19. Cirera, J.; Paesani, F. Theoretical prediction of spin-crossover temperatures in ligand-driven light-induced spin change systems. Inorg. Chem. 2012, 51, 8194-8201.

20. Swart, M. Accurate spin-state energies for iron complexes. J. Chem. Theory Comput. 2008, 4, 2057-2066.

21. Ye, S.; Neese, F. Accurate modeling of spin-state energetics in spin-crossover systems with modern density functional theory. Inorg. Chem. 2010, 49, 772-774.

22. Fouqueau, A.; Casida, M.E.; Lawson, L.M.; Hauser, A.; Neese, F. Comparison of density functionals for energy and structural differences between the high- $\left[{ }^{5} \mathrm{~T}_{2 g}:\left(\mathrm{t}_{2 g}^{4}\right)\left(\mathrm{e}_{g}^{2}\right)\right]$ and low- $\left[{ }^{1} \mathrm{~A}_{1 g}:\left(\mathrm{t}_{2 g}^{6}\right)\left(\mathrm{e}_{g}^{0}\right)\right]$ spin states of iron(II) coordination compounds: II. Comparison of results for more than ten modern functionals with ligand field theory and $\mathrm{Ab}$ initio results for hexaquoferrous dication, $\left[\mathrm{Fe}(\mathrm{H} 2 \mathrm{O})_{6}\right]^{2+}$ and hexaminoferrous dication $\left[\mathrm{Fe}\left(\mathrm{NH}_{3}\right)_{6}\right]^{2+}$. J. Chem. Phys. 2005, 122, 044110.

23. Fouqueau, A.; Mer, S.; Casida, M.E.; Daku, L.M.L.; Hauser, A.; Mineva, T.; Neese, F. Comparison of density funtionals for energy and structural differences between the high- $\left[{ }^{5} \mathrm{~T}_{2 g}:\left(\mathrm{t}_{2 g}\right)^{4}\left(\mathrm{e}_{g}\right)^{2}\right]$ and low$\left[{ }^{1} \mathrm{~A}_{1 g}:\left(\mathrm{t}_{2 g}\right)^{6}\left(\mathrm{e}_{g}\right)^{0}\right]$ spin states of the hexaquoferrous cation $\left[\mathrm{Fe}\left(\mathrm{H}_{2} \mathrm{O}\right)_{6}\right]^{2+}$. J. Chem. Phys. 2004, 120, 9473-9486.

24. Lawson Daku, L.M.; Vargas, A.; Hauser, A.; Fouqueau, A.; Casida, M.E. Assessment of density functionals for the high-spin/low-spin energy difference in the low-spin iron(II) tris(2,2'-bipyridine) complex. ChemPhysChem. 2005, 6, 1393-1410.

25. Ganzenmuller, G.; Berkaine, N.; Fouqueau, A.; Casida, M.E.; Reiher, M. Comparison of density functionals for differences between the high- $\left({ }^{5} \mathrm{~T}_{2 g}\right)$ and low- $\left({ }^{1} \mathrm{~A}_{1 g}\right)$ spin states of iron(II) compounds. IV. Results for the ferrous complexes [Fe(L)('NHS $\left.\left.{ }_{4}^{\prime}\right)\right]$. J. Chem. Phys. 2005, 122, 234321.

26. Slimani, A.; Yu, X.; Muraoka, A.; Boukheddaden, K.; Yamashita, K. Reparametrization approach of DFT functionals based on the equilibrium temperature of spin-crossover compounds. J. Phys. Chem. A 2014, 118, 9005-9012.

27. Paulsen, H.; Duelund, L.; Zimmermann, A.; Averseng, F.; Gerdan, M.; Winkler, H.; Toftlund, H.; Trautwein, A.X. Substituent effects on the spin-transition temperature in complexes with tris(pyrazolyl) ligands. Monatshefte Chem. 2003, 134, 295-306.

28. Paulsen, H.; Trautwein, A.X. Calculation of the electronic energy differences of spin crossover complexes. J. Phys. Chem. Solids 2004, 65, 793-798.

29. Güell, M.; Sola, M.; Swart, M. Spin-state splittings of iron(II) complexes with trispyrazolyl ligands. Polyhedron 2010, 29, 84-93.

30. Le Guennic, B.; Matouzenko, G.S.; Borshch, S.A. Topology of spin-crossover polymers and mutual influence of ligands. Eur. J. Inorg. Chem. 2008, 2008, 3020-3023.

31. Kepenekian, M.; Guennic, B.L.; Robert, V. Primary role of the electrostatic contributions in a rational growth of hysteresis loop in spin-crossover Fe(II) complexes. J. Am. Chem. Soc. 2009, 131, 11498-11502.

32. Matouzenko, G.S.; Perrin, M.; Guennic, B.L.; Genre, C.; Molnar, G.; Bousseksou, A.; Borshch, S.A. Spin crossover behavior in a family of iron(II) zigzag chain coordination polymers. Dalton Trans. 2007, doi:10.1039/B615410G.

33. Rackwitz, S.; Klopper, W.; Schunemann, V.; Wolny, J.A. Quantification of intramolecular cooperativity in polynuclear spin crossover Fe(II) complexes by density functional theory calculations. Phys. Chem. Chem. Phys. 2013, 15, 15450-15458.

34. Zein, S.; Borshch, S.A. Energetics of binuclear spin transition complexes. J. Am. Chem. Soc. 2005, 127, 16197-16201.

35. Ruiz, E. Charge transport properties of spin crossover systems. Phys. Chem. Chem. Phys. 2014, 16, 14-22.

36. Cirera, J.; Ruiz, E. Theoretical modeling of two-step spin-crossover transitions in $\mathrm{Fe}^{I I}$ dinuclear systems. J. Mater. Chem. C 2015, 3, 7954-7961.

37. Saha-Dasgupta, T.; Oppeneer, P.M. Computational design of magnetic metal-organic complexes and coordination polymers with spin-switchable functionalities. MRS Bull. 2014, 39, 614-620.

38. Lemercier, G.; Brefuel, N.; Shova, S.; Wolny, J.A.; Dahan, F.; Verelst, M.; Paulsen, H.; Trautwein, A.X.; Tuchagues, J.P. A range of spin-crossover temperature $\mathrm{T}_{1 / 2}>300 \mathrm{~K}$ results from out-of-sphere anion exchange in a series of ferrous materials based on the 4-(4-Imidazolylmethyl)-2-(2-imidazolylmethyl) imidazole (trim) ligand, $\left[\mathrm{Fe}(\text { trim })_{2}\right] \mathrm{X}_{2}(\mathrm{X}=\mathrm{F}, \mathrm{Cl}, \mathrm{Br}, \mathrm{I})$ : Comparison of experimental results with those derived from density functional theory calculations. Chem. Eur. J. 2006, 12, 7421-7432. 
39. Middlemiss, D.S.; Portinari, D.; Grey, C.P.; Morrison, C.A.; Wilson, C.C. Spin crossover in the $\mathrm{CsFe}^{\mathrm{II}}\left[\mathrm{Cr}_{6}^{\mathrm{III}(\mathrm{CN})}\right]$ Prussian blue analog: Phonons and thermodynamics from hybrid functionals. Phys. Rev. B 2010, 81, doi:10.1103/PhysRevB.81.184410.

40. Cirera, J.; Babin, V.; Paesani, F. Theoretical modeling of spin crossover in metal-organic frameworks: $\left[\mathrm{Fe}(\mathrm{pz})_{2} \mathrm{Pt}(\mathrm{CN})_{4}\right]$ as a case study. Inorg. Chem. 2014, 53, 11020-11028.

41. Middlemiss, D.S.; Deeth, R.J. First principles calculation of a large variation in dielectric tensor through the spin crossover in the $\mathrm{CsFe}\left[\mathrm{Cr}(\mathrm{CN})_{6}\right]$ Prussian blue analogue. J. Chem. Phys. 2014, 140, 144503.

42. Lebègue, S.; Pillet, S.; Ángyán, J.G. Modeling spin-crossover compounds by periodic DFT+U approach. Phys. Rev. B 2008, 78, doi:10.1103/PhysRevB.78.024433.

43. Matar, S.F.; Létard, J.F. Ab initio molecular and solid-state studies of the spin crossover system [Fe(phen $\left.)_{2}(\mathrm{NCS})_{2}\right]$. Z. Naturforsch. B 2010, 65, 565-570.

44. Zhang, Y. Predicting critical temperatures of iron(II) spin crossover materials: Density functional theory plus $U$ approach. J. Chem. Phys. 2014, 141, 214703.

45. Chen, J.; Millis, A.J.; Marianetti, C.A. Density functional plus dynamical mean-field theory of the spin-crossover molecule Fe(phen) ${ }_{2}(\mathrm{NCS})_{2}$. Phys. Rev. B 2015, 91, doi:10.1103/PhysRevB.91.241111.

46. König, E.; Madeja, K. ${ }^{5} \mathrm{~T}_{2}{ }^{1} \mathrm{~A}_{1}$ Equilibriums in some iron(II)-bis(1,10-phenanthroline) complexes. Inorg. Chem. 1967, 6, 48-55.

47. König, E.; Madeja, K. Correction. Equilibria in some iron(II)-bis(1,10-phenanthroline) complexes. Inorg. Chem. 1968, 7, 2677.

48. Gallois, B.; Real, J.A.; Hauw, C.; Zarembowitch, J. Structural changes associated with the spin transition in $\mathrm{Fe}(\mathrm{phen})_{2}(\mathrm{NCS})_{2}$ : A single-crystal X-ray investigation. Inorg. Chem. 1990, 29, 1152-1158.

49. Legrand, V.; Pillet, S.; Weber, H.P.; Souhassou, M.; Létard, J.F.; Guionneau, P.; Lecomte, C. On the precision and accuracy of structural analysis of light-induced metastable states. J. Appl. Cryst. 2007, 40, 1076-1088.

50. Bousseksou, A.; McGarvey, J.J.; Varret, F.; Real, J.A.; Tuchagues, J.P.; Dennis, A.C.; Boillot, M.L. Raman spectroscopy of the high- and low-spin states of the spin crossover complex Fe(phen $)_{2}(\mathrm{NCS})_{2}$ : An initial approach to estimation of vibrational contributions to the associated entropy change. Chem. Phys. Lett. 2000, 318, 409-416.

51. Palfi, V.K.; Guillona, T.; Paulsen, H.; Molnar, G.; Bousseksou, A. Isotope effects on the vibrational spectra of the $\mathrm{Fe}(\text { Phen })_{2}(\mathrm{NCS})_{2}$ spin-crossover complex studied by density functional calculation. C. R. Chim. 2005, $8,1317-1325$.

52. Sinitskiy, A.V.; Tchougréeff, A.L.; Dronskowski, R. Phenomenological model of spin crossover in molecular crystals as derived from atom-atom potentials. Phys. Chem. Chem. Phys. 2011, 13, 13238-13246.

53. Sorai, M.; Seki, S. Phonon coupled cooperative low-spin ${ }^{1} \mathrm{~A}_{1}$ high-spin ${ }^{5} \mathrm{~T}_{2}$ transition in $\left[\mathrm{Fe}(\mathrm{phen})_{2}(\mathrm{NCS})_{2}\right]$ and $\left[\mathrm{Fe}(\text { phen })_{2}(\mathrm{NCSe})_{2}\right]$ crystals. J. Phys. Chem. Solids 1974, 35, 555-570.

54. Grimme, S. Semiempirical GGA-type density functional constructed with a long-range dispersion correction. J. Comp. Chem. 2006, 27, 1787-1799.

55. Harvey, J.N. On the accuracy of density functional theory in transition metal chemistry. Annu. Rep. Prog. Chem. 2006, 102, 203-226.

56. Chiruta, D.; Linares, J.; Garcia, Y.; Dahoo, P.R.; Dimian, M. Analysis of the hysteretic behaviour of 3D spin crossover compounds by using an ising-like model. Eur. J. Inorg. Chem. 2013, 2013, 3601-3608.

57. Mebs, S.; Braun, B.; Kositzki, R.; Limberg, C.; Haumann, M. Abrupt versus gradual spin-crossover in $\mathrm{Fe}^{\mathrm{II}}$ (phen $)_{2}(\mathrm{NCS})_{2}$ and $\mathrm{Fe}^{\mathrm{III}}$ (dedtc) $)_{3}$ compared by X-ray absorption and emission spectroscopy and quantum-chemical calculations. Inorg. Chem. 2015, 54, 11606-11624.

58. Giannozzi, P.; Baroni, S.; Bonini, N.; Calandra, M.; Car, R.; Cavazzoni, C.; Ceresoli, D.; Chiarotti, G.L.; Cococcioni, M.; Dabo, I.; et al. QUANTUM ESPRESSO: A modular and open-source software project for quantum simulations of materials. J. Phys. Condens. Matter 2009, 21, doi:10.1088/0953-8984/21/39/395502.

59. Perdew, J.P.; Zunger, A. Self-interaction correction to density-functional approximations for many-electron systems. Phys. Rev. B 1981, 23, 5048-5079.

60. Vanderbilt, D. Soft self-consistent pseudopotentials in a generalized eigenvalue formalism. Phys. Rev. B 1990, 41, 7892-7895.

61. Pseudopotentials Fe.pz-sp-van_ak.upf, S.pz-van_ak.upf, N.pz-van_ak.upf, C.pz-van_ak.upf and H.pz-van_ak.upf. Available online: www.quantum-espresso.org/pseudopotentials (accessed on 10 September 2015). 
62. Cococcioni, M.; de Gironcoli, S. Linear response approach to the calculation of the effective interaction parameters in the LDA + U method. Phys. Rev. B 2005, 71, doi:10.1103/PhysRevB.71.035105.

(c) 2016 by the author; licensee MDPI, Basel, Switzerland. This article is an open access article distributed under the terms and conditions of the Creative Commons by Attribution (CC-BY) license (http://creativecommons.org/licenses/by/4.0/). 